\title{
Alteration of Mevalonate Pathway Related Enzyme Expressions in Pressure Overload- Induced Cardiac Hypertrophy and Associated Heart Failure with Preserved Ejection Fraction
}

\author{
Bin Chen ${ }^{\mathrm{a}}$ Li-Yun Zhong ${ }^{\mathrm{b}}$ Jin-Xiu Yang ${ }^{\mathrm{c}}$ Yan-Yun Pan ${ }^{\mathrm{a}}$ Fei Chen ${ }^{\mathrm{a}}$ Jian Yang ${ }^{\mathrm{a}}$ Tao Wu \\ Shen-Jiang $\mathrm{Hu}^{\mathrm{a}}$ \\ aInstitute of Cardiology, The First Affiliated Hospital, College of Medicine, Zhejiang University, \\ 'Department of Ultrasound, The First Affiliated Hospital, College of Medicine, Zhejiang University, \\ 'Department of Cardiology, Zhejiang Traditional Chinese Medical Hospital, Hangzhou, China
}

\section{Key Words}

Pressure overload $\cdot$ Cardiac hypertrophy $\cdot$ Diastolic dysfunction $\cdot$ Mevalonate pathway $\cdot$ Small GTP-binding proteins

\begin{abstract}
Background: Abnormalities of the mevalonate pathway, an important cellular metabolic pathway, are common in many diseases including cardiovascular disease. The mevalonate pathway related enzyme expressions in pressure overload-induced cardiac hypertrophy and associated diastolic dysfunction remains largely unknown. This study aims to investigate whether the expression of mevalonate pathway related enzyme is altered during the progression of cardiac hypertrophy and associated diastolic dysfunction induced by pressure overload. Methods: Male Sprague-Dawley (SD) rats were randomly divided into two groups: the suprarenal abdominal aortic coarctation (AAC) group and the sham group. Results: Histological and echocardiographic assessments showed that there was a significant cardiovascular remodeling in the AAC group compared with the sham group after 3 weeks post-operatively, and the left ventricular (LV) diastolic function was reduced at 8 and 14 weeks post-operatively in the AAC group, without any change in systolic function during the study. The tissue of the heart and the abdominal aorta proximal to the coarctation showed over-expression of several enzymes, including 3-hydroxy-3-methylglutaryl coenzyme A reductase (HMGR), farnesyl diphosphate synthase (FDPS), farnesyltransferase- $\alpha$ (FNTA), farnesyltransferase- $\beta$ (FNTB), geranylgeranyltransferase type I (GGTase-I) and the activation of their downstream proteins was enhanced. Conclusions: AAC induced compensatory LV hypertrophy to decompensatory diastolic dysfunction, accompanied by altered expression of several key enzymes in the mevalonate pathway.
\end{abstract}

Copyright (C) 2013 S. Karger AG, Basel 


\section{Introduction}

Heart failure with preserved ejection fraction (HFpEF) is the clinical syndrome of heart failure characterized by impaired diastolic function but normal systolic function [1]. HFpEF, which has the same rate of morbidity and hospitalization as heart failure with reduced ejection fraction (HFrEF), accounts for approximately $40 \%$ of heart failure cases overall [2, 3]. Although initially thought to have a superior prognosis, patients with HFpEF are now known to have as poor a prognosis as those patients with HFrEF [4]. With advancements in modern heart failure treatment, the prognosis for patients with HFrEF has improved over the last two decades; however, this is not the case for patients with HFpEF [4]. The most common systemic disease associated with HFpEF is hypertension. Diastolic dysfunction is present in half of patients with hypertension, which along with other stimuli causes an increase in the cardiac mass as well as the reconfiguration of myocardiac proteins $[4,5]$.

One study found that pitavastatin had a beneficial effect on LV diastolic function [6]. Additionally, several studies have found a survival benefit in patients with HFpEF who are receiving statin therapy [7-9], which mainly exerts a competitive inhibition on HMGR in the mevalonate pathway. The mevalonate pathway plays an important role in many cellular functions, including control of cell growth and differentiation by the synthesis of sterol isoprenoids such as cholesterol and non-sterol isoprenoids such as farnesyl pyrophosphate (FPP) and geranylgeranyl pyrophosphate (GGPP) [10]. Isoprenoids influence the activity of small G proteins such as Ras and Rho through prenylation [11]. Furthermore, treatment with statins before the initiation of LV hypertrophy has been reported to suppress the development of LV hypertrophy [12-14].

However, as the mechanism of HFpEF is still not fully understood, the prognosis for these patients remains poor. Although patients with HFpEF may yield benefits from statin therapy, the role of mevalonate pathway related enzymes in pressure overload-induced cardiac hypertrophy and associated diastolic dysfunction remains largely unknown. Therefore, the aim of this study was to investigate whether changes in the expression of mevalonate pathway related enzymes, such as 3-hydroxy-3-methylglutaryl-coenzyme A reductase (HMGR), phospho-3-hydroxy-3-methylglutaryl-coenzyme A reductase (P-HMGR), farnesyl diphosphate synthase (FDPS), squalene synthase (SQS), farnesyltransferase $\alpha$ (FNTA), farnesyltransferase $\beta$ (FNTB) and geranylgeranyltransferase type I (GGTase-I) and its downstream small G proteins (Ras, RhoA, Rac, Cdc42) during the progression of cardiac hypertrophy and associated diastolic dysfunction induced by pressure overload (Fig.1A).

\section{Materials and Methods}

\section{Animal Preparation}

All animal studies were approved by the Institutional Council for Animal Research of Zhejiang University and performed according to the guidelines for the Care and Use of Laboratory Animals published by the US National Institutes of Health (NIH Publication No. 85-23, revised 1996). HFpEF was induced by suprarenal abdominal aortic coarctation in male Sprague-Dawley (SD) rats (190-210 grams). After rats in the AAC group were anesthetized, the suprarenal portion of the aorta was exposed and a blunted 22-gauge needle was placed adjacent to the aorta. A ligature (4-0 silk) was snugly tied around both the aorta and the needle. The needle was then removed, leaving the internal diameter of the aorta approximately equal to that of the needle. A sham operation was performed in the same manner in the sham group, without constriction of the abdominal aorta. The animals were housed under controlled environmental conditions and given free access to water and regular chow.

Assessment of Cardiac Hypertrophy and Function by Echocardiography

Rats were evaluated at 3, 5, 8 and 14 weeks post-operatively. After rats were anesthetized, imaging was performed with rats placed in the left lateral decubitus position using a Vivid 7 (GE Vingmed, Horten, Norway) echocardiography machine with a $14 \mathrm{MHz}$ phased array probe. M-mode imaging of the long axis view 
Chen et al.: Potential Therapeutic Targets for the Management of Refractory Diastolic Dysfunction and HFpEF

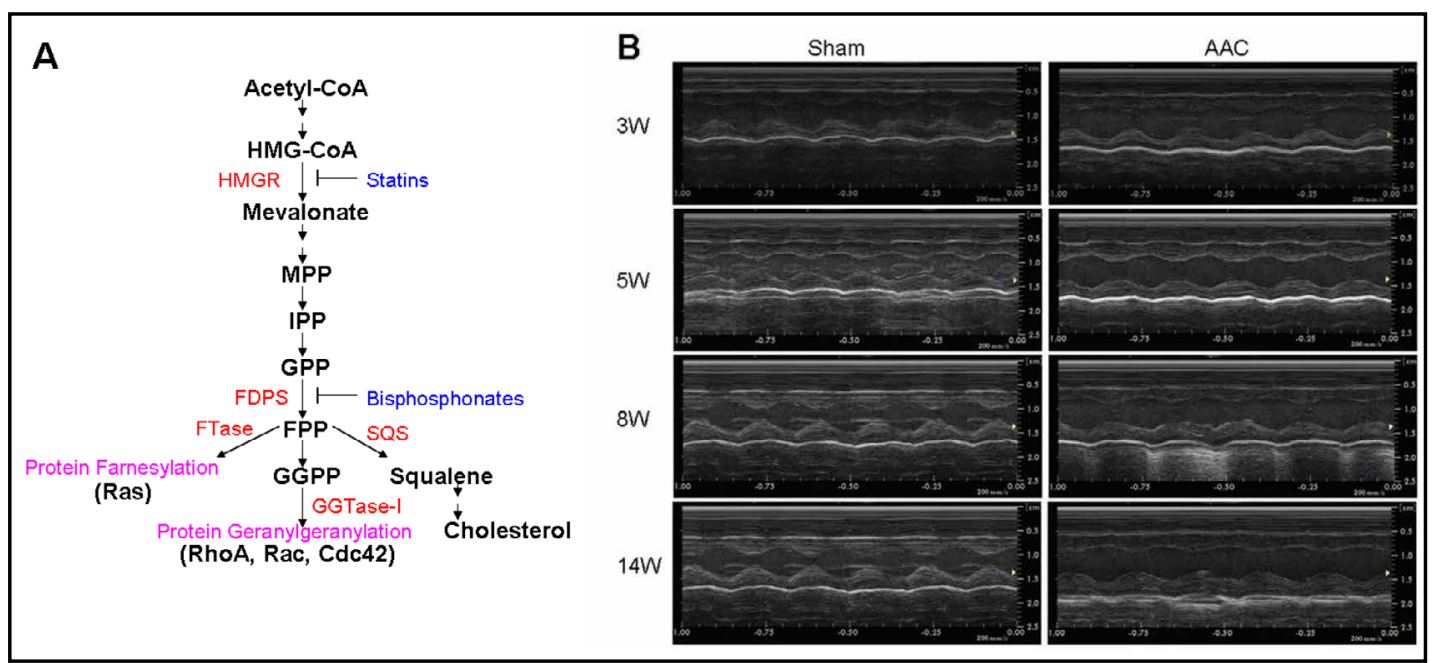

Fig. 1. (A) Schematic diagram of the mevalonate pathway. MPP, mevalonate pyrophosphate; IPP, isopentenyl pyrophosphate; GPP, geranyl pyrophosphate; FPP, farnesyl pyrophosphate; GGPP, geranylgeranyl pyrophosphate; HMGR, 3-hydroxy-3-methylglutaryl CoA reductase; FDPS, farnesyl diphosphate synthase; FTase, farnesyltransferase; GGTase-I, geranylgeranyltransferase type I; SQS, squalene synthase. (B) Representative M-mode echocardiography in the sham and AAC groups at 3, 5, 8 and 14 weeks post-operatively.

was taken to measure the LV internal diameter at end-systole and the LV internal diameter at end-diastole (LVIDs and LVIDd). The interventricular septum wall thickness and posterior wall thickness at the end of the diastolic phase (IVSd and LVPWd) were also measured. The ejection fraction (EF) and LV shortening fraction (FS) were then calculated using the echocardiographic system. A two-dimensional, apical, four-chamber view allowed for measurement of $\mathrm{LV}$ inflow peak velocities at both early and late filling stages (E-wave peak velocity and A-wave peak velocity) by pulse wave Doppler. The E/A ratio was then calculated. The mitral valve (MV) inflow E wave deceleration time (DT) was also measured. All measurements were averaged for three consecutive beats.

\section{Hemodynamic Measurement}

Afterechocardiographicrecording, hemodynamicstudies were performed using the methods previously described at 3, 5, 8 and 14 weeks post-operatively [15]. Biological signal acquisition and processing system (MedLab-U/4C501H, Nanjing medease science and technology Co., Ltd, China) and software (MedLabV6.3, Nanjing medease science and technology Co., Ltd, China) were used to get hemodynamic data. Briefly, after rats were anesthetized, a polyethylene tube (PE50, Becton-Dickinson) was introduced via the right carotid artery to measure the systolic blood pressure (SBP), diastolic blood pressure (DBP), pulse pressure (PP) and mean arterial pressure (MAP). The tube was then further advanced into the LV to measure the heart rate (HR), LV end-diastolic pressure (LVEDP) and LV systolic pressure (LVSP), as well as the maximal rate of rise and decline of $\mathrm{LV}$ pressure $( \pm \mathrm{dP} / \mathrm{dt}$ max). The tube was withdrawn and the incision of the right carotid artery was gripped with an artery clip. The tube was then inserted into the abdominal artery distal to the abdominal aortic coarctation to abtain SBP, DBP, PP and MAP measurements.

\section{Brain Natriuretic Peptide Measurement}

After the hemodynamic studies were completed, a $4 \mathrm{ml}$ blood sample was taken. The plasma was isolated and the level of brain natriuretic peptide (BNP) was measured using a commercially available enzyme immunoassay (EIA) kit (Peninsula Laboratories, Bachem Group, USA).

\section{Histological Analysis}

After the blood sample was collected, the heart was quickly excised, and the LV and the right ventricle were separated and weighed. The lungs were also quickly excised and weighed. Sections of the LV free wall and septum were fixed in 10\% neutral formalin, embedded in paraffin, and sequentially stained with hematoxylin-eosin and Masson's trichrome. The entire aorta was removed, and two small sections of aortic 
Chen et al.: Potential Therapeutic Targets for the Management of Refractory Diastolic Dysfunction and HFpEF

\begin{tabular}{rcccccccc}
\hline Week & SBP(mmHg) & DBP(mmHg) & PP(mmHg) & MAP(mmHg) & $+\mathrm{dP} / \mathrm{dt}(\mathrm{mmHg} / \mathrm{s})$ & -dP/dt(mmHg/s) & LVEDP(mmHg) & LVSP(mmHg) \\
\hline Sham & & & & & & & & \\
$3(\mathrm{n}=6)$ & $141 \pm 4$ & $113 \pm 6$ & $29 \pm 2$ & $122 \pm 6$ & $5908 \pm 213$ & $4223 \pm 280$ & $4.9 \pm 0.5$ & $142 \pm 5$ \\
$5(\mathrm{n}=6)$ & $125 \pm 6$ & $102 \pm 8$ & $23 \pm 1$ & $106 \pm 7$ & $6521 \pm 402$ & $4602 \pm 365$ & $5.1 \pm 0.6$ & $130 \pm 4$ \\
$8(\mathrm{n}=6)$ & $134 \pm 3$ & $99 \pm 5$ & $30 \pm 3$ & $109 \pm 4$ & $5786 \pm 367$ & $4277 \pm 279$ & $5.4 \pm 0.4$ & $139 \pm 3$ \\
$14(\mathrm{n}=6)$ & $136 \pm 5$ & $90 \pm 6$ & $40 \pm 2$ & $104 \pm 5$ & $5566 \pm 321$ & $4000 \pm 345$ & $6.0 \pm 0.8$ & $140 \pm 4$ \\
AAC & & & & & & & & \\
$3(\mathrm{n}=6)$ & $222 \pm 11^{*}$ & $150 \pm 8^{*}$ & $80 \pm 9^{*}$ & $178 \pm 8^{*}$ & $10021 \pm 745^{*}$ & $7541 \pm 524^{*}$ & $4.4 \pm 0.6$ & $213 \pm 5^{*}$ \\
$5(\mathrm{n}=6)$ & $198 \pm 10^{*}$ & $149 \pm 9^{*}$ & $54 \pm 2^{*}$ & $155 \pm 9^{*}$ & $10547 \pm 656^{*}$ & $7854 \pm 397 *$ & $6.9 \pm 0.5$ & $203 \pm 5^{*}$ \\
$8(\mathrm{n}=6)$ & $212 \pm 13^{*}$ & $166 \pm 9^{*}$ & $67 \pm 3^{*}$ & $187 \pm 9^{*}$ & $7528 \pm 235^{*}$ & $6301 \pm 656^{*}$ & $11.5 \pm 0.9 *$ & $216 \pm 9^{*}$ \\
$14(\mathrm{n}=6)$ & $186 \pm 12^{*}$ & $148 \pm 7^{*}$ & $50 \pm 3^{*}$ & $152 \pm 7^{*}$ & $5535 \pm 256$ & $4231 \pm 342$ & $16.6 \pm 1.1 *$ & $190 \pm 8^{*}$ \\
\hline
\end{tabular}

Table 1. Hemodynamic Variables in Sham-Operated and Abdominal Aortic Coarctation Rats at 3, 5, 8 and 14 Weeks. Data are given as mean \pm SEM. n, no. of experiments. SBP, systolic blood pressure; DBP, diastolic blood pressure; PP, pulse pressure; MAP, mean artery pressure; $+\mathrm{dP} / \mathrm{dt}$ and $-\mathrm{dP} / \mathrm{dt}$, maximal rate of rise and decline of left ventricular pressure respectively; LVEDP, left ventricular end diastolic pressure; LVESP, left ventricular end systolic pressure. ${ }^{*} \mathrm{P}<0.01$ vs. agematched Sham; ${ }^{*} \mathrm{P}<0.05$ vs. agematched Sham.

rings on either side of the coarctation were obtained, fixed in 10\% neutral formalin, embedded in paraffin, and stained with hematoxylin-eosin and Masson's trichrome. The rest of the heart and aortic rings were collected immediately and stored at $-80^{\circ} \mathrm{C}$ for subsequent analysis.

\section{Western blot analysis}

Total proteins were isolated from the heart, aorta and liver tissue, and protein concentrations were determined using the BCA method. Proteins $(60 \mu \mathrm{g})$ were separated in polyacryl-amide gels and transferred onto a polyvinylidene difluoride membrane (Millipore, MA, USA). After blocking, the membranes were incubated with antibodies to HMGR (ab98018, Abcam, UK), P-HMGR (ab78275, Abcam, UK), FDPS (ab38854, Abcam, UK), FNTA (\#3335-1, Epitomics, USA), FNTB (ab74206, Abcam, UK), SQS (ab71637, Abcam, UK), GGTase-I (ab122122, Abcam, UK), P-ERK1/2 (\#4370, Cell Signaling, USA), ERK1/2 (\#4695, Cell Signaling, USA), RhoA (\#2117, Cell Signaling, USA), Ras (\#3233-1, Epitomics, USA) and GAPDH (ab9485, Abcam, UK). Hybridizing bands were visualized by the enhanced chemiluminescence method. To ensure equal protein loading, GAPDH was used as an endogenous control.

Activation of RhoA, Rac1, Cdc42, and Ras

RhoA, Rac1, Cdc42, and Ras activity was determined by an absorbance-based G-LISA Activation Assay Biochemistry kit (Kit \#BK124; \#BK128; \#BK127; \#BK131; Cytoskeleton, Denver, CO, USA) according to manufacturer instructions.

Statistical analysis

Results are expressed as mean \pm SEM. One-way ANOVA followed by Bonferroni post hoc test was used to determine significant differences among 3 groups, SPSS version 16.0 (SPSS, Chicago, IL, USA) was used for all analyses. $\mathrm{P}<0.05$ was considered significant.

\section{Results}

Blood Pressure

Hemodynamic parameters such as SBP, DBP, PP and MAP were all greatly elevated in the AAC group compared with the sham group at 3, 5, 8 and 14 weeks post-operatively (Table 
Chen et al.: Potential Therapeutic Targets for the Management of Refractory Diastolic Dysfunction and HFpEF

\begin{tabular}{|c|c|c|c|c|c|c|c|c|}
\hline Week & IVSd $(\mathrm{mm})$ & LVPWd (mm) & LVIDd (mm) & LVIDs (mm) & $\mathrm{EF}(\%)$ & FS $(\%)$ & E/A ratio & $\mathrm{DT}(\mathrm{ms})$ \\
\hline \multicolumn{9}{|l|}{ Sham } \\
\hline $0(n=9)$ & $0.78 \pm 0.02$ & $0.80 \pm 0.01$ & $4.98 \pm 0.13$ & $2.88 \pm 0.16$ & $78.1 \pm 1.3$ & $42.1 \pm 0.5$ & $1.82 \pm 0.06$ & $39 \pm 1.8$ \\
\hline $3(n=6)$ & $1.05 \pm 0.03$ & $1.02 \pm 0.04$ & $5.43 \pm 0.14$ & $3.15 \pm 0.12$ & $75.9 \pm 1.4$ & $41.8 \pm 0.8$ & $1.67 \pm 0.09$ & $40 \pm 2.2$ \\
\hline $5(n=6)$ & $1.26 \pm 0.05$ & $1.26 \pm 0.01$ & $5.58 \pm 0.17$ & $3.21 \pm 0.12$ & $77.5 \pm 1.5$ & $41.4 \pm 1.4$ & $1.74 \pm 0.06$ & $36 \pm 2.5$ \\
\hline $8(n=6)$ & $1.34 \pm 0.03$ & $1.42 \pm 0.04$ & $5.72 \pm 0.13$ & $3.32 \pm 0.14$ & $78.8 \pm 1.3$ & $42.1 \pm 0.9$ & $1.63 \pm 0.07$ & $37 \pm 1.7$ \\
\hline $14(n=6)$ & $1.51 \pm 0.04$ & $1.72 \pm 0.03$ & $6.01 \pm 0.19$ & $3.52 \pm 0.13$ & $76.1 \pm 1.6$ & $41.3 \pm 1.3$ & $1.55 \pm 0.03$ & $38 \pm 1.9$ \\
\hline \multicolumn{9}{|l|}{ AAC } \\
\hline $0(n=9)$ & $0.79 \pm 0.01$ & $0.81 \pm 0.02$ & $5.01 \pm 0.12$ & $3.03 \pm 0.15$ & $78.3 \pm 1.4$ & $41.2 \pm 0.9$ & $1.89 \pm 0.06$ & $38 \pm 1.9$ \\
\hline $3(n=6)$ & $1.21 \pm 0.01^{*}$ & $1.22 \pm 0.02 *$ & $5.23 \pm 0.21$ & $2.76 \pm 0.12$ & $80.8 \pm 1.0^{\#}$ & $46.2 \pm 1.1^{\#}$ & $1.75 \pm 0.08$ & $37 \pm 2.1$ \\
\hline $5(n=6)$ & $1.42 \pm 0.01^{*}$ & $1.44 \pm 0.02 *$ & $6.11 \pm 0.12$ & $3.55 \pm 0.07$ & $76.8 \pm 1.1$ & $40.7 \pm 1.0$ & $1.76 \pm 0.04$ & $39 \pm 2.2$ \\
\hline $8(n=6)$ & $1.58 \pm 0.03^{*}$ & $1.71 \pm 0.04^{*}$ & $6.24 \pm 0.15$ & $3.67 \pm 0.11$ & $79.5 \pm 1.9$ & $41.3 \pm 1.3$ & $1.31 \pm 0.05^{\#}$ & $46 \pm 2.2^{\#}$ \\
\hline $14(n=6)$ & $1.71 \pm 0.07 *$ & $2.15 \pm 0.06^{*}$ & $6.41 \pm 0.14$ & $3.86 \pm 0.12$ & $75.5 \pm 1.5$ & $40.2 \pm 1.2$ & $1.22 \pm 0.07^{\#}$ & $55 \pm 2.8^{*}$ \\
\hline
\end{tabular}

Table 2. Echocardiographic Variables in Sham-Operated and Abdominal Aortic Coarctation Rats at 0, 3, 5, 8 and 14 Weeks. Data are given as mean \pm SEM. n, no. of experiments. IVSd, interventricular septum thickness in end-diastole; LVPWd, left ventricular posterior wall thickness in end-diastole; LVIDd, left ventricular internal dimension in end-diastole; LVIDs, left ventricular internal dimension in end-systole; EF, ejection fraction; FS, fractional shortening; E/A, ratio of early to late diastolic filling velocities; DT, deceleration time of early diastolic filling. ${ }^{*} \mathrm{P}<0.01$ vs. age-matched Sham; ${ }^{\#} \mathrm{P}<0.05$ vs. age-matched Sham.

1). The SBP gradient between the carotid and abdominal aorta distal to the coarctation was approximately $70 \mathrm{mmHg}$ in the AAC group and $10 \mathrm{mmHg}$ in the sham group (Data not shown).

\section{Cardiac and Abdominal Aortic Remodeling}

Baseline echocardiographic findings were similar in the AAC and sham groups (Table 2). There was a significant increase in diastolic wall thicknesses (IVSd and LVPWd) at the end of 3, 5, 8 and 14 weeks post-operatively in the AAC group compared with the sham group ( $\mathrm{P}<0.01$, Table 2 and Fig. $1 \mathrm{~B}$ ). LV weight and the ratio of LV weight to body weight were significantly increased in the AAC group $(\mathrm{P}<0.01$, Table 3$)$. Representative sections of hematoxylin-eosin-stained heart tissue are shown in Fig. 2A. The myocyte cross-sectional area was significantly increased in the AAC group compared with the sham group at 3, 5, 8 and 14 weeks post-operatively (Fig. 2B). Representative Mallory-Azan stained sections of the LV myocardium are shown in Fig. 2C. Progressive perivascular fibrosis in the myocardium was observed in the AAC group: it increased by $100 \%$ at 3 weeks $(\mathrm{P}<0.01)$ and by $600 \%$ at 14 weeks $(\mathrm{P}<0.01)$ compared with the sham group. A summary of the perivascular fibrosis data is shown in Fig. 2D.

The AAC group demonstrated obvious abdominal aortic remodeling (Fig. 3A). The medial thickness in the abdominal aortic segment proximal to the coarctation increased by approximately 35\% compared with that in the sham group at 3, 5 and 8 weeks postoperatively, reaching approximately a $60 \%$ increase at 14 weeks post-operatively (Fig. $3 \mathrm{~B})$. In the AAC group, the medial thickness in the abdominal aortic segment distal to the coarctation was reduced compared with the segment proximal to the coarctation. The distal segment in the AAC group was also thinner than the same segment in the sham group at 5 and 8 weeks post-operatively (Fig. 3B).

The LV Systolic and Diastolic Function

The systolic function measured by FS was higher in the AAC group than the sham group at 3 weeks post-operatively (FS, $41.8 \pm 0.8$ in sham vs. $46.2 \pm 1.1$ in $A A C, P<0.05$, Table 2 ). There 


\begin{tabular}{lccccccc}
\hline Week & BW(g) & LVW(g) & LVW/BW(mg/g) & RVW(g) & RVW/BW(mg/g) & LW(g) & LW/BW(mg/g) \\
\hline Sham & & & & & & \\
3(n=6) & $327 \pm 7$ & $0.73 \pm 0.03$ & $2.20 \pm 0.09$ & $0.19 \pm 0.01$ & $0.58 \pm 0.02$ & $1.59 \pm 0.10$ & $4.81 \pm 0.34$ \\
$5(\mathrm{n}=6)$ & $420 \pm 7$ & $0.87 \pm 0.04$ & $2.11 \pm 0.13$ & $0.22 \pm 0.01$ & $0.53 \pm 0.01$ & $1.71 \pm 0.14$ & $4.01 \pm 0.30$ \\
$8(\mathrm{n}=6)$ & $446 \pm 6$ & $0.90 \pm 0.01$ & $2.06 \pm 0.06$ & $0.23 \pm 0.01$ & $0.52 \pm 0.01$ & $1.77 \pm 0.11$ & $3.94 \pm 0.19$ \\
$14(\mathrm{n}=6)$ & $560 \pm 12$ & $1.11 \pm 0.05$ & $1.95 \pm 0.07$ & $0.25 \pm 0.01$ & $0.45 \pm 0.02$ & $1.91 \pm 0.07$ & $3.41 \pm 0.12$ \\
AAC & & & & & & & 4.12 \\
$3(\mathrm{n}=6)$ & $326 \pm 6$ & $1.11 \pm 0.06^{*}$ & $3.37 \pm 0.11^{*}$ & $0.19 \pm 0.01$ & $0.58 \pm 0.03$ & $1.46 \pm 0.12$ & $4.65 \pm 0.29$ \\
$5(\mathrm{n}=6)$ & $400 \pm 11$ & $1.23 \pm 0.03^{*}$ & $3.15 \pm 0.25^{*}$ & $0.23 \pm 0.02$ & $0.57 \pm 0.04$ & $1.73 \pm 0.13$ & $4.45 \pm 0.31$ \\
$8(\mathrm{n}=6)$ & $443 \pm 5$ & $1.36 \pm 0.09^{*}$ & $3.10 \pm 0.12^{*}$ & $0.25 \pm 0.01$ & $0.56 \pm 0.02$ & $1.80 \pm 0.12$ & $4.07 \pm 0.26$ \\
$14(\mathrm{n}=6)$ & $530 \pm 8$ & $1.62 \pm 0.05^{*}$ & $3.06 \pm 0.14^{*}$ & $0.27 \pm 0.01$ & $0.51 \pm 0.02$ & $2.22 \pm 0.09$ & $4.11 \pm 0.22^{\#}$ \\
\hline
\end{tabular}

Table 3. Organ weight Variables in Sham-Operated and Abdominal Aortic Coarctation Rats at 3, 5, 8 and 14 Weeks. Data are given as mean \pm SEM. n, no. of experiments. BW, body weight; LVW, left ventricular weight; RVW, right ventricular weight; LW, lung weight. ${ }^{*} \mathrm{P}<0.01$ vs. age-matched $\mathrm{Sham}$; $\mathrm{P}<0.05$ vs. agematched Sham

was no statistical difference between the two groups at 5, 8 and 14 weeks. The variation of EF was the same as that of FS $(\mathrm{P}<0.05$, Table 2$)$. Carotid catheterization showed that the maximal rate of rise of $\mathrm{LV}$ pressure $(+\mathrm{dP} / \mathrm{dt})$ was almost twofold elevated in the AAC group compared with the sham group at 3 and 5 weeks post-operatively $(+\mathrm{dP} / \mathrm{dt} \mathrm{mmHg} / \mathrm{s}$ : sham, $5908 \pm 213$, AAC, $10021 \pm 745, \mathrm{P}<0.01 ;+\mathrm{dP} / \mathrm{dt} \mathrm{mmHg} / \mathrm{s}$ : sham, 6521 \pm 402 , AAC, $10547 \pm 656$, $\mathrm{P}<0.01$; Table 1). Although the $+\mathrm{dP} / \mathrm{dt}$ began to decrease in the AAC group at 8 weeks postoperatively compared with the same measurement at 3 and 5 weeks post-operatively, it was still not lowered compared with the sham group (Table 1).

LV diastolic dysfunction first appeared at 8 weeks post-operatively. Parameters of diastolic function (E/A and DT) showed a significant difference between the AAC and sham groups at 8 and 14 weeks post-operatively (Table 2). Hemodynamic parameters showed that the maximal rate of decline of $\mathrm{LV}$ pressure $(-\mathrm{dP} / \mathrm{dt})$ in the AAC group began to decline at 8 weeks post-operatively, declining to near the level in the sham group at 14 weeks postoperatively (Table 1), although it initially rose at 3 and 5 weeks post-operatively (Table 1). The rise of LVEDP at 8 and 14 weeks $(\mathrm{P}<0.01$, Table 1$)$ and the ratio of lung weight to body weight at 14 weeks ( $\mathrm{P}<0.05$, Table 3$)$ were further indications of the LV diastolic dysfunction. Increased ventricular expression of the genes encoding for BNP is a hallmark change of cardiac hypertrophy and diastolic dysfunction [16]. We found that the levels of plasma BNP in the AAC group were significantly higher than those in the sham group (Fig. 3C).

\section{HMGR and FDPS Expression Levels}

HMGR, the rate-limiting enzyme for the mevalonate pathway, is one of the most intensively investigated proteins in biochemistry. We found that the expression of HMGR was significantly higher in the heart tissue of the AAC group compared with the sham group, while the expression of P-HMGR was similar in the two groups (Fig. 4A and Fig. 4C). However, HMGR and P-HMGR showed no change in the abdominal aorta and liver tissue of the AAC group (Data not shown). FDPS is a key enzyme in the isoprene biosynthetic pathway and directly catalyzes the formation of FPP and GPP [17]. Immunoblots demonstrated that the level of FDPS expression was slightly higher in the heart and abdominal aorta tissue of the AAC group compared with the sham group (Fig. 4A, Fig. 4B, Fig. 4C and Fig.4D). 


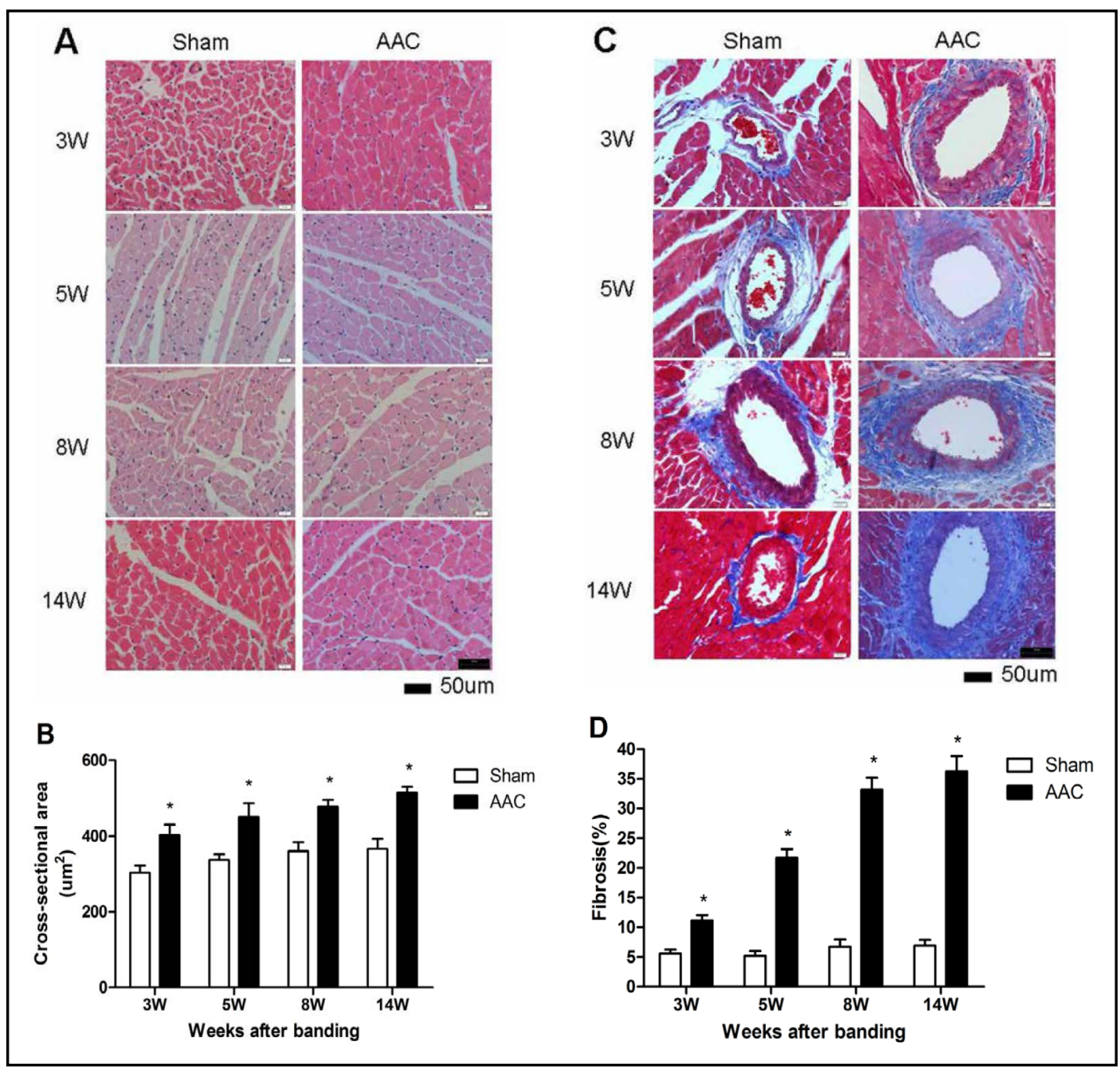

Fig. 2. (A) Representative microphotographs of hematoxylin-eosin-stained transverse sections of the left ventricular myocardium in the sham and AAC groups at 3, 5, 8 and 14 weeks (W) post-operatively (400x). (B) Cross-sectional area of cardiomyocytes in the sham and AAC groups at 3, 5, 8 and 14 weeks (W) post-operatively. Data were obtained from 6 rats for each group, and at least 200 cardiomyocytes were analyzed for each rat, ${ }^{*} \mathrm{P}<0.01$ vs. age-matched sham. (C) Representative Mallory-Azan-stained sections of the left ventricular myocardium in the sham and AAC groups at 3, 5, 8 and 14 weeks (W) post-operatively (400x). The AAC group showed marked perivascular fibrosis (stained blue) and extended to the intermuscular interstitial spaces. (D) The Mallory-Azan-stained fibrotic area (stained blue) fraction in the sham and AAC groups at 3, 5, 8 and 14 weeks (W) post-operatively. ${ }^{*} \mathrm{P}<0.01$ vs. age-matched sham.

\section{GGTase-I, FNTA, FNTB and SQS Expression Levels}

After being synthesized by FDPS, FPP participates in three biological processes, biosynthesis of sterol catalyzed by SQS, farnesylation of Ras by FTase (FTase: FNTA and FNTB), and geranylgeranylation of Rho by GGTase-I [18]. SQS expression did not differ in the heart and aorta tissue between the AAC group and the sham group (Fig. 4A). The results of immunoblots demonstrated greater levels of FNTA and FNTB in tissue from the heart and abdominal aorta proximal to the coarctation of the AAC group compared with tissue from the sham group (Fig. 4A, Fig. 4B, Fig. 4C and Fig. 4D). The expression of GGTase-I was increased in the tissue from heart and abdominal aorta proximal to the coarctation in the AAC group at 14 weeks post-operatively (Fig. 4A, Fig. 4B, Fig. 4C and Fig. 4D), while the expression of 


\section{Cellular Physiology $\quad$ Cell Physiol Biochem 2013;32:1761-1775 and Biochemistry \\ Chen et al.: Potential Therapeutic Targets for the Management of Refractory Diastolic Dysfunction and HFpEF}

Fig. 3. (A) Representative microphotographs of hematoxylin-eosin-stained transverse sections of the abdominal aorta proximal and distal to the coarctation in the sham and AAC groups at $3,5,8$ and 14 weeks (W) post-operatively $(400 \times)$. Sham, abdominal aorta close to the coarctation in the sham group; ACC P, abdominal aorta proximal to the coarctation in the AAC group; ACC D, abdominal aorta distal to the coarctation in the AAC group. (B) The abdominal aortic medial thickness proximal and distal to the coarctation in the sham and AAC groups at 3, 5, 8 and 14 weeks (W) post-operatively. Data were obtained from 6 rats for each group. ${ }^{*} \mathrm{P}<$ 0.01 vs. age-matched sham, ${ }^{* *} \mathrm{P}<0.01$ vs. age-

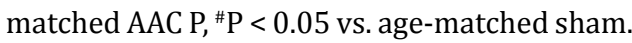
Sham, abdominal aorta close to the coarctation in the sham group; AAC P, abdominal aorta proximal to the coarctation in the AAC group; AAC $D$, abdominal aorta distal to the coarctation in the AAC group. (C) Plasma BNP in the sham and AAC groups. ${ }^{*} \mathrm{P}<0.01$ vs. age-matched sham.

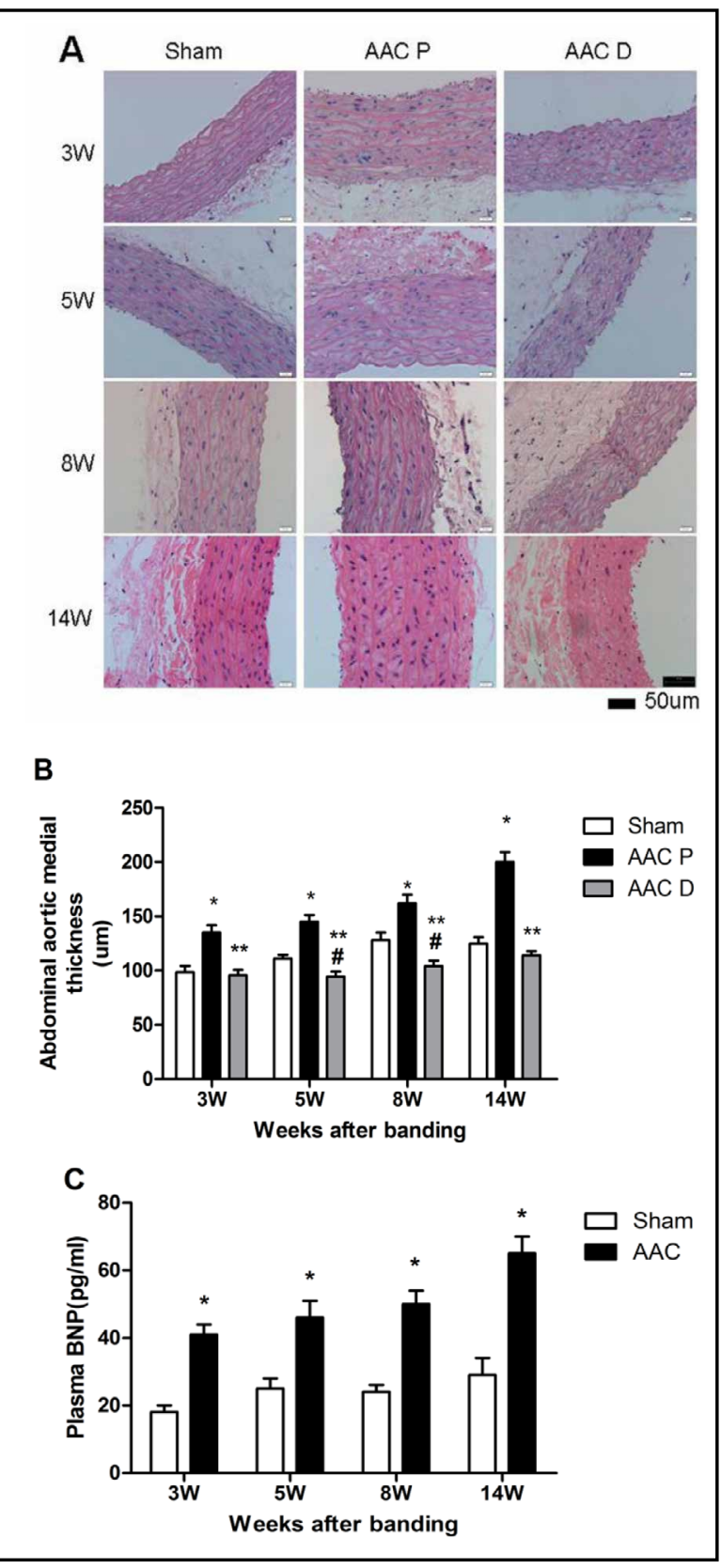

GGTase-I was significantly reduced in the abdominal aorta distal to the coarctation in the AAC group compared with the sham group at all ages tested (Fig. 4B and Fig. 4D).

\section{The Small G Protein, ERK1/2 Expression Levels and Activity}

Total Ras, RhoA, and ERK1/2 protein levels in the heart and aorta did not differ between the sham group and the AAC group (Fig. 4A and Fig. 4B). A significant Ras/ERK1/2 activation was found in the heart and abdominal aorta tissue in the AAC group compared with the sham group at all ages tested (Fig. 4A, Fig. 4B, Fig. 4C, Fig. 4D, Fig. 5A and Fig. 5B). We found only slight RhoA activation in the tissue of the heart and abdominal aorta proximal to the coarctation in the AAC group at 14 weeks post-operatively (Fig. 5A and Fig. 5B). However, activation of Cdc42 and Rac1 was not found in the heart and aorta tissue in the AAC group (Fig. 5A and Fig. 5B). 


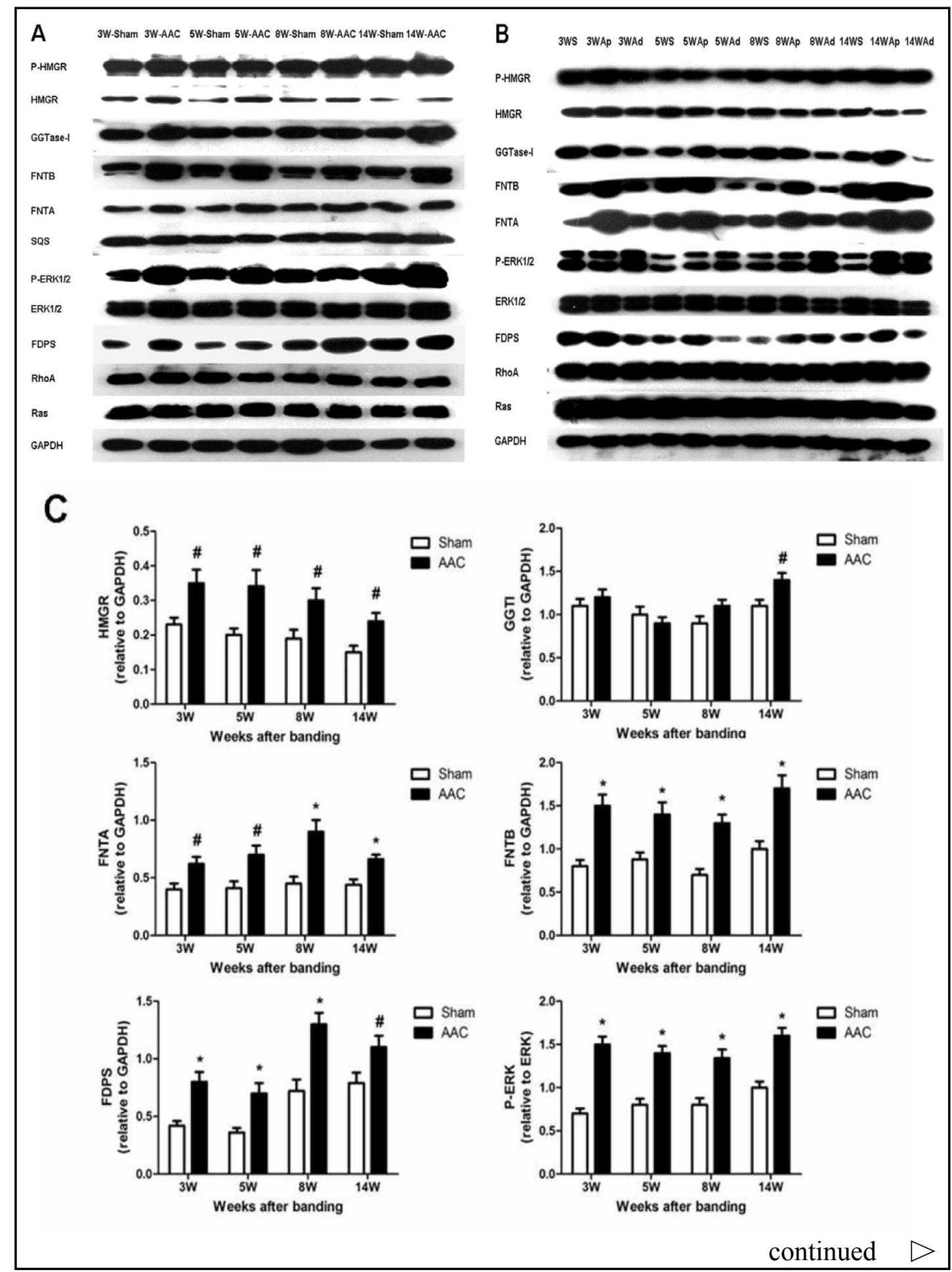

\section{Discussion}

There is currently no effective therapy for HFpEF, which is highly prevalent in community and is associated with significant morbidity and mortality [19]. Diastolic dysfunction is of uncertain clinical relevance, but it is a known harbinger of HFpEF. We used a well-validated animal model of diastolic dysfunction, the cornerstone of HFpEF, to study alterations of mevalonate pathway-related enzyme expression in this condition. To our knowledge, this 
D
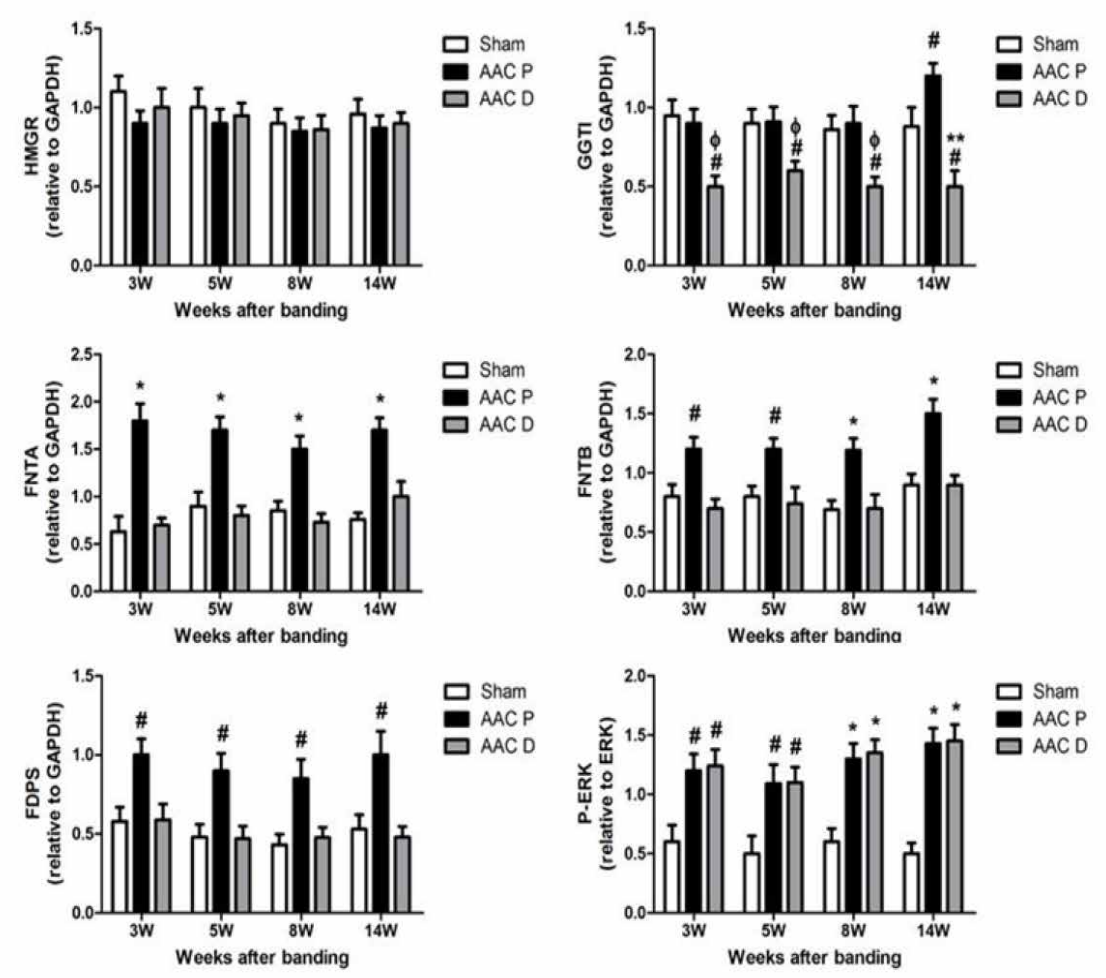

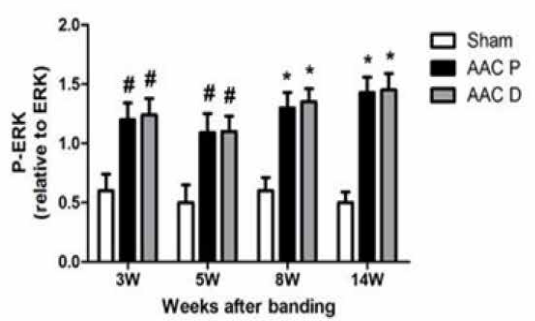

Fig. 4. (A) Proteins were extracted from the left ventricular myocardium in the sham and AAC groups at 3,5, 8 and 14 weeks post-operatively. The expression of P-HMGR, HMGR, GGTase-I, FNTB, FNTA, SQS, P-ERK1/2, ERK1/2, FDPS, RhoA and Ras was quantified by western blot analysis. Equal loading was validated by the expression of GAPDH. (B) Proteins were extracted from the abdominal aorta in the sham and AAC groups at 3, 5, 8 and 14 weeks post-operatively. The expression of GGTase-I, FNTB, FNTA, P-ERK1/2, ERK1/2, FDPS, RhoA and Ras was quantified by western blot analysis. Equal loading was validated by the expression of GAPDH. WS, weeks, the abdominal aorta close to the coarctation in the sham group; WAp, weeks, the abdominal aorta proximal to the coarctation in the AAC group; WAd, weeks, the abdominal aorta distal to the coarctation in the AAC group. (C) The western blot results of the proteins from left ventricular myocardium were quantified by densitometry. The band density of HMGR, GGTase-I, FNTA, FNTB and FDPS was normalized to the band density of GAPDH, and the band density of P-ERK1/2 was normalized to the band density of ERK1/2. Data are expressed as mean $\pm \mathrm{SEM}, \mathrm{n}=6$. ${ }^{*} \mathrm{P}<0.01$ vs. age-matched sham, ${ }^{*} \mathrm{P}<0.05$ vs. age-matched sham. (D) The western blot results of the proteins from abdominal aorta were quantified by densitometry. The band density of HMGR, GGTase-I, FNTA, FNTB and FDPS was normalized to the band density of GAPDH, and the band density of P-ERK1/2 was normalized to the band density of ERK1/2. Data are expressed as mean $\pm \mathrm{SEM}, \mathrm{n}=6 .{ }^{*} \mathrm{P}<0.01$ vs. age-matched sham, ${ }^{*} \mathrm{P}<0.05$ vs. age-matched sham. ${ }^{* *} \mathrm{P}<$ 0.01 vs. age-matched AAC $P,{ }^{\Phi} \mathrm{P}<0.05$ vs. age-matched AAC P. Sham, abdominal aorta close to the coarctation in the sham group; AAC P, abdominal aorta proximal to the coarctation in the AAC group; AAC D, abdominal aorta distal to the coarctation in the AAC group.

is the first study to examine alteration of the expression of mevalonate pathway related enzymes from compensatory cardiac hypertrophy to diastolic dysfunction. The cardiac diastolic dysfunction in the AAC group did not appear until 8 and 14 weeks post-operatively, with a rise in LVEDP and DT, a drop in E/A, and the emergence of pulmonary edema at 14 weeks in the AAC group. LV and abdominal aortic remodeling occurred with preserved $\mathrm{EF}$ and FS at 3, 5, 8 and 14 weeks post-operatively in the AAC group.

We found that the level of FNTA and FNTB protein were higher in the tissue of the hypertrophic $\mathrm{LV}$ and the abdominal aorta proximal to the coarctation in the AAC group 


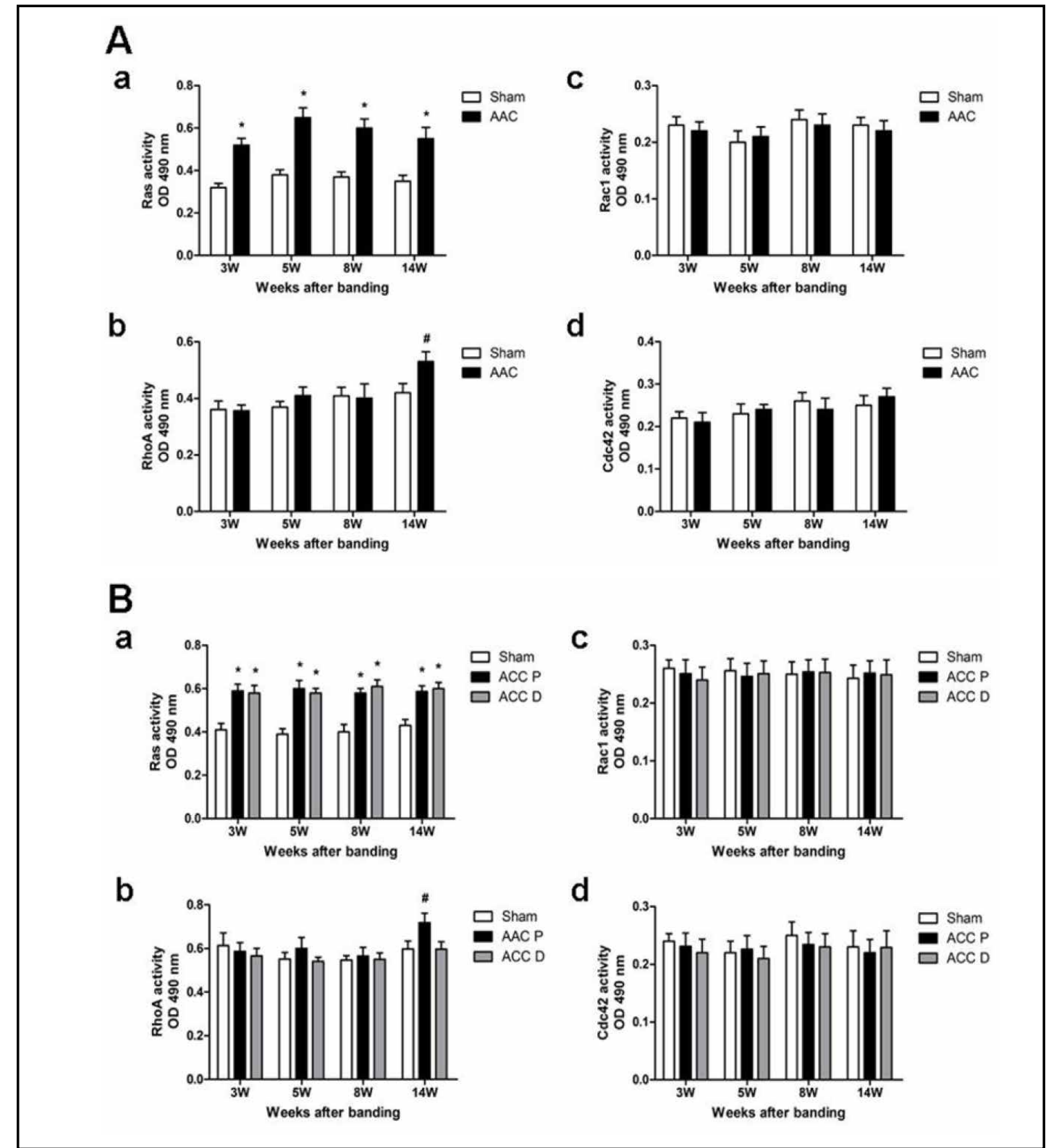

Fig. 5. The activation of Ras, RhoA, Rac1, and Cdc42 was showed in the figure $a, b, c$ and d respectively. (A) Proteins were extracted from the left ventricular myocardium in the sham and AAC groups at 3, 5, 8 and 14 weeks post-operatively. ${ }^{*} \mathrm{P}<0.01$ vs. age-matched sham, ${ }^{\#} \mathrm{P}<0.05$ vs. age-matched sham. (B) Proteins were extracted from the abdominal aorta in the sham and AAC groups at 3, 5, 8 and 14 weeks post-operatively. ${ }^{*} \mathrm{P}<0.01$ vs. age-matched sham, ${ }^{\mathrm{P}}<0.05$ vs. age-matched sham. Sham, abdominal aorta close to the coarctation in the sham group; AAC P, abdominal aorta proximal to the coarctation in the AAC group; AAC D, abdominal aorta distal to the coarctation in the AAC group.

compared with the sham group. Ras translocation from the cytoplasm to the plasma membrane is dependent on farnesylation by FTase [18], thus, the increased level of FTase catalyzed more farnesylation of Ras in the AAC group than in the sham group. This result corresponded with a significant Ras activation in the hypertrophic LV and abdominal aorta proximal to the coarctation at 3, 5, 8 and 14 weeks post-operatively. Cardiovascular remodeling has been implicated in the activity of several small GTPases. Ras, the first small $\mathrm{G}$ protein shown to be involved in cardiovascular remodeling, induces a significant increase 
Chen et al.: Potential Therapeutic Targets for the Management of Refractory Diastolic Dysfunction and HFpEF

in cardiac mass and vascular smooth muscle cell (VSMC) proliferation [20-23]. Ras activates a kinase cascade involving Raf, mitogen-activated protein kinases (MAPK) and extracellular signal-regulated kinase (ERK) phosphorylates, which in turn induce cell proliferation and growth regulation that lead to cardiovascular remodeling [24, 25]. Our data confirm and further extend these results. Significant ERK1/2 activation was found in the hypertrophic myocardium and abdominal aorta tissue at 3, 5, 8 and 14 weeks post-operatively, which is consistent with the result of another study [26]. While FTase inhibitors attenuate some of the transcriptional changes in hypertrophic myocytes that are induced by norepinephrine or endothelin-1 (ET-1) [27]. Thus we believe that the elevated expression of FTase in the AAC group may be associated with cardiovascular remodeling in this model.

GGTase-I was elevated in the tissue of the hypertrophic LV and the abdominal aorta proximal to the coarctation at 14 weeks in the AAC group. The biological activity of Rho GTPases (RhoA, Rac1, Cdc42) depends on the process of geranylgeranylation by GGTase-I [18]. Thus, elevated GGTase-I levels lead to increased activation of Rho GTPases. However in our experimental model, we only found RhoA activation in the tissue of the hypertrophic $\mathrm{LV}$ and abdominal aorta proximal to the coarctation at 14 weeks post-operatively. The small GTPases are a point of convergence of several pathways important in cardiovascular remodeling, including those stimulated by Angiotensin II (AngII), mechanical stress, and growth factors $[28,29]$, while AngII inhibition reduces cardiovascular remodeling induced by pressure overload via inhibiting ERK1/2 activiation [26]. Unexpectedly, GGTase-I was suppressed in the atrophic aorta distal to the coarctation, providing further evidence that GGTase-I may play an important role in cardiovascular remodeling.

Increased expression of HMGR and FDPS was found in the hypertrophic LV tissue in the AAC group compared with the sham group at 3, 5, 8 and 14 weeks post-operatively. Simvastatin significantly reduces hypertrophy in rats with pressure overload due to aortic banding [30]. It has been previously demonstrated that selective inhibition of FTase blocks Ras processing and that the inhibition of HMGR interferes with Ras anchorage to cell membrane and its activation by reducing the supply of farnesyl groups $[10,31,32]$. However, the expression of HMGR in the abdominal aorta was not increased in the AAC group. Studies have indicated that the enzymes of non-sterol pathways generally have higher affinities than those of sterol pathways for mevalonate-derived substrates [33]. This may lead to enough toxic accumulation of some intermediates, including FPP and GGPP, despite of the similar level of HMGR expression, which may cause the pathologic cardiovascular remodeling. Statin inhibition of HMGR, the rate-controlling step for polyprenoid and cholesterol biosynthesis, is a less specific way of reducing Ras farnesylation. However, statins reduce the stimulation of protein accumulation by AngII in cardiac myocytes, and importantly, the inhibition can be prevented by mevalonate [34]. FDPS is another key enzyme in the mevalonate pathway, and it catalyzes the formation of FPP and GGPP [17]. With the rise of FDPS in the myocardium and abdominal aorta proximal to the coarctation, there would be increased accumulation of FPP and GGPP, leading to increased isoprenylation of small GTPases. Our previous study found that inhibition of FDPS attenuated cardiac hypertrophy and fibrosis in spontaneously hypertensive rats [35]. Thus it could be inferred that cardiovascular remodeling is associated with increased expression of HMGR and FDPS in this model.

It has been shown that the cardiac renin-angiotensin system (RAS) is associated with the cardiac hypertrophy induced by pressure overload [36]. Previous studies strongly suggest Ang II induce the elevation of FPPS in cardiac hypertrophy [37, 38]. It may infer that the mevalonate pathway is activated partly by pressure overload through the RAS in our model, meanwhile other mechanism like mechanical stretch may also play a role [39].

Activation of Ras and ERK1/2 were almost identical on either side of the coarctation in our study. AngII, which is the principal mediator of the physiological actions of the RAS, has a long-term effect involving the activation of the Ras signaling cascade [40]. Therefore activation of Ras and ERK1/2 in the abdominal aorta distal to the coarctation may be associated with AngII, which may be paracrined by the cells in the abdominal aorta proximal to the coarctation. Although activation of Ras and ERK1/2 was similar at either side of 
the coarctation, the hypertrophy was only in the aorta proximal to the coarctation but not distal. A possible explanation for this finding is that many factors participate in the process of cardiovascular remodeling, including mechanical stimulation and activation of proteins associated with cardiovascular reconfiguration. Furthermore, it is possible that the elevated enzymes in the mevalonate pathway may influence cardiovascular remodeling through other pathways in addition to the small GTPases.

Previous studies found that HMGR inhibitors inhibited Ras signaling and prevented the LV hypertrophy induced by AAC and the Ras-dependent transition from compensatory LV hypertrophy to diastolic dysfunction $[23,41]$. Our study found more conclusive data and further focused on upstream and downstream components of the Ras pathway. In addition to HMGR and FDPS, we also detected no difference between the AAC and sham groups with regards to FNTA, FNTB, GGTase-I, Ras, RhoA, ERK1/2 and ERK1/2 phosphorylation in liver. We also found that the expression of SQS in heart, aorta and liver tissue was not elevated in the AAC group. This finding implies that cholesterol may not play a role in this model.

Although we didn't examine whether inhibition of mevalonate pathway attenuated cardiac hypertrophy and diastolic dysfunction induced by pressure overload, it is reasonable to infer that the mevalonate pathway plays an important role in pressure-overload model. Firstly, our previous study found that the expression of key enzymes in the mevalonate pathway in the heart and aorta was raise in spontaneously hypertensive rats (SHR) [42], and inhibition of FDPS attenuated cardiac hypertrophy and fibrosis in SHR [43]. Secondly, the antihypertrophic mechanism of statins may involve inhibition of the mevalonate pathway $[30,44]$, and statins prevented LV hypertrophy development in pressure-overload animals through inhibiting Ras signaling [41]. Meanwhile, Ventricular expression of ras fusion gene induced cardiac hypertrophy and selective diastolic dysfunction in transgenic mice [23], and mechanical stretch-induced cardiac hypertrophy was linked to mevalonate pathway [39].

In conclusion, the data presented in this study document that pressure overload induced compensatory cardiac hypertrophy to decompensatory diastolic dysfunction, and the alteration of the expression of key enzymes in the mevalonate pathway in the heart and aorta is implicated in the process of cardiovascular remodeling and diastolic dysfunction, likely through the Ras/MAPK/ERK pathway. Our findings indicate that the manipulation of key enzymes and downstream small G proteins in the mevalonate pathway may open the field for novel therapies, offering many potential therapeutic targets for the management of refractory diastolic dysfunction and HFpEF in the future.

\section{Acknowledgements}

This work was supported by the National Natural Sciences Foundation of China (Project Nos. 30470715, 30870939 and 81170242 ).

\section{Conflict of Interests}

The authors confirm that there are no conflicts of interest.

\section{References}

1 Vasan RS, Levy D: Defining diastolic heart failure: a call for standardized diagnostic criteria. Circulation 2000;101:2118-2121.

2 Senni M, Redfield MM: Heart failure with preserved systolic function. A different natural history? J Am Coll Cardiol 2001;38:1277-1282.

3 Tsutsui H, Tsuchihashi M, Takeshita A: Mortality and readmission of hospitalized patients with congestive heart failure and preserved versus depressed systolic function. Am J Cardiol 2001;88:530-533. 
Chen et al.: Potential Therapeutic Targets for the Management of Refractory Diastolic Dysfunction and HFpEF

4 Owan TE, Hodge DO, Herges RM, Jacobsen SJ, Roger VL, Redfield MM: Trends in prevalence and outcome of heart failure with preserved ejection fraction. N Engl J Med 2006;355:251-259.

-5 Solomon SD, Verma A, Desai A, Hassanein A, Izzo J, Oparil S, Lacourciere Y, Lee J, Seifu Y, Hilkert RJ, Rocha R, Pitt B: Effect of intensive versus standard blood pressure lowering on diastolic function in patients with uncontrolled hypertension and diastolic dysfunction. Hypertension 2010;55:241-248.

6 Warita S, Kawasaki M, Tanaka R, Ono K, Kojima T, Hirose T, Iwama M, Watanabe T, Nishigaki K, Takemura G, Noda T, Watanabe S, Minatoguchi S: Effects of pitavastatin on cardiac structure and function and on prevention of atrial fibrillation in elderly hypertensive patients: a prospective study of 2-years' follow-up. Circ J 2012;76:2755-2762.

7 Fukuta H, Sane DC, Brucks S, Little WC: Statin therapy may be associated with lower mortality in patients with diastolic heart failure: a preliminary report. Circulation 2005;112:357-363.

8 Gomez-Soto FM, Romero SP, Bernal JA, Escobar MA, Puerto JL, Andrey JL, Ruiz P, Gomez F: Mortality and morbidity of newly diagnosed heart failure treated with statins: a propensity-adjusted cohort study. Int J Cardiol 2010;140:210-218.

-9 Paulus WJ, van Ballegoij JJ: Treatment of heart failure with normal ejection fraction: an inconvenient truth! J Am Coll Cardiol 2010;55:526-537.

10 Goldstein JL, Brown MS: Regulation of the mevalonate pathway. Nature 1990;343:425-430.

11 Casey PJ: Protein lipidation in cell signaling. Science 1995;268:221-225.

12 Takemoto M, Node K, Nakagami H, Liao Y, Grimm M, Takemoto Y, Kitakaze M, Liao JK: Statins as antioxidant therapy for preventing cardiac myocyte hypertrophy. J Clin Invest 2001;108:1429-1437.

13 Hasegawa H, Yamamoto R, Takano H, Mizukami M, Asakawa M, Nagai T, Komuro I: 3-Hydroxy-3methylglutaryl coenzyme A reductase inhibitors prevent the development of cardiac hypertrophy and heart failure in rats. J Mol Cell Cardiol 2003;35:953-960.

-14 Hayashidani S, Tsutsui H, Shiomi T, Suematsu N, Kinugawa S, Ide T, Wen J, Takeshita A: Fluvastatin, a 3-hydroxy-3-methylglutaryl coenzyme a reductase inhibitor, attenuates left ventricular remodeling and failure after experimental myocardial infarction. Circulation 2002;105:868-873.

15 Sun YL, Hu SJ, Wang LH, Hu Y, Zhou JY: Effect of beta-blockers on cardiac function and calcium handling protein in postinfarction heart failure rats. Chest 2005;128:1812-1821.

16 Nishikimi T: Do plasma levels of brain natriuretic peptide (BNP) and N-terminal proBNP (NT-proBNP) increase in diastolic dysfunction as well as in systolic dysfunction? Circ J 2012;76:2540-2541.

17 Szkopinska A, Plochocka D: Farnesyl diphosphate synthase; regulation of product specificity. Acta Biochim Pol 2005;52:45-55.

18 Takai Y, Sasaki T, Matozaki T: Small GTP-binding proteins. Physiol Rev 2001;81:153-208.

19 Hogg K, Swedberg K, McMurray J: Heart failure with preserved left ventricular systolic function; epidemiology, clinical characteristics, and prognosis. J Am Coll Cardiol 2004;43:317-327.

20 Abdellatif M, Packer SE, Michael LH, Zhang D, Charng MJ, Schneider MD: A Ras-dependent pathway regulates RNA polymerase II phosphorylation in cardiac myocytes: implications for cardiac hypertrophy. Mol Cell Biol 1998;18:6729-6736.

21 Chien KR, Hoshijima M: Unravelling Ras signals in cardiovascular disease. Nat Cell Biol 2004;6:807-808.

22 Chen KH, Guo X, Ma D, Guo Y, Li Q, Yang D, Li P, Qiu X, Wen S, Xiao RP, Tang J: Dysregulation of HSG triggers vascular proliferative disorders. Nat Cell Biol 2004;6:872-883.

23 Hunter JJ, Tanaka N, Rockman HA, Ross J Jr, Chien KR: Ventricular expression of a MLC-2v-ras fusion gene induces cardiac hypertrophy and selective diastolic dysfunction in transgenic mice. J Biol Chem 1995;270:23173-23178.

24 Klemke RL, Cai S, Giannini AL, Gallagher PJ, de Lanerolle P, Cheresh DA: Regulation of cell motility by mitogen-activated protein kinase. J Cell Biol 1997;137:481-492.

25 Hunter JJ, Chien KR: Signaling pathways for cardiac hypertrophy and failure. N Engl J Med 1999;341:12761283.

26 Curcio A, Torella D, Iaconetti C, Pasceri E, Sabatino J, Sorrentino S, Giampa S, Micieli M, Polimeni A, Henning BJ, Leone A, Catalucci D, Ellison GM, Condorelli G, Indolfi C: MicroRNA-1 downregulation increases connexin 43 displacement and induces ventricular tachyarrhythmias in rodent hypertrophic hearts. PLoS One 2013;8:e70158.

27 Calderone A, Abdelaziz N, Colombo F, Schreiber KL, Rindt H: A farnesyltransferase inhibitor attenuates cardiac myocyte hypertrophy and gene expression. J Mol Cell Cardiol 2000;32:1127-1140. 
Chen et al.: Potential Therapeutic Targets for the Management of Refractory Diastolic Dysfunction and HFpEF

28 Sah VP, Hoshijima M, Chien KR, Brown JH: Rho is required for Galphaq and alpha1-adrenergic receptor signaling in cardiomyocytes. Dissociation of Ras and Rho pathways. J Biol Chem 1996;271:31185-31190.

29 Aikawa R, Komuro I, Nagai R, Yazaki Y: Rho plays an important role in angiotensin II-induced hypertrophic responses in cardiac myocytes. Mol Cell Biochem 2000;212:177-182.

-30 Luo JD, Zhang WW, Zhang GP, Guan JX, Chen X: Simvastatin inhibits cardiac hypertrophy and angiotensinconverting enzyme activity in rats with aortic stenosis. Clin Exp Pharmacol Physiol 1999;26:903-908.

- 31 Gibbs JB, Pompliano DL, Mosser SD, Rands E, Lingham RB, Singh SB, Scolnick EM, Kohl NE, Oliff A: Selective inhibition of farnesyl-protein transferase blocks ras processing in vivo. J Biol Chem 1993;268:7617-7620.

32 Laufs U, Marra D, Node K, Liao JK: 3-Hydroxy-3-methylglutaryl-CoA reductase inhibitors attenuate vascular smooth muscle proliferation by preventing rho GTPase-induced down-regulation of p27(Kip1). J Biol Chem 1999;274:21926-21931.

33 Brown MS, Goldstein JL: Multivalent feedback regulation of HMG CoA reductase, a control mechanism coordinating isoprenoid synthesis and cell growth. J Lipid Res 1980;21:505-517.

34 Oi S, Haneda T, Osaki J, Kashiwagi Y, Nakamura Y, Kawabe J, Kikuchi K: Lovastatin prevents angiotensin IIinduced cardiac hypertrophy in cultured neonatal rat heart cells. Eur J Pharmacol 1999;376:139-148.

-35 Chen GP, Li L, Yang Y, Fu M, Yao L, Wu T, Zhang XQ, Hu SJ: Chronic inhibition of farnesyl pyrophosphate synthase improves endothelial function in spontaneously hypertensive rats. Biochem Pharmacol 2010;80:1684-1689.

-36 Baker KM, Booz GW, Dostal DE: Cardiac actions of angiotensin II: Role of an intracardiac renin-angiotensin system. Annu Rev Physiol 1992;54:227-241.

-37 Li L, Yi-Ming W, Li ZZ, Zhao L, Yu YS, Li DJ, Xia CY, Liu JG, Su DF: Local RAS and inflammatory factors are involved in cardiovascular hypertrophy in spontaneously hypertensive rats. Pharmacol Res 2008;58:196201.

-38 Ye Y, Mou Y, Bai B, Li L, Chen GP, Hu SJ: Knockdown of farnesylpyrophosphate synthase prevents angiotensin II-mediated cardiac hypertrophy. Int J Biochem Cell Biol 2010;42:2056-2064.

39 Kashiwagi Y, Haneda T, Osaki J, Miyata S, Kikuchi K: Mechanical stretch activates a pathway linked to mevalonate metabolism in cultured neonatal rat heart cells. Hypertens Res 1998;21:109-119.

40 Guo DF, Sun YL, Hamet P, Inagami T: The angiotensin II type 1 receptor and receptor-associated proteins. Cell Res 2001;11:165-180.

41 Indolfi C, Di Lorenzo E, Perrino C, Stingone AM, Curcio A, Torella D, Cittadini A, Cardone L, Coppola C, Cavuto L, Arcucci O, Sacca L, Avvedimento EV, Chiariello M: Hydroxymethylglutaryl coenzyme A reductase inhibitor simvastatin prevents cardiac hypertrophy induced by pressure overload and inhibits p21ras activation. Circulation 2002;106:2118-2124.

42 Han J, Jiang D-M, Du C-Q, Hu S-J: Alteration of Enzyme Expressions in Mevalonate Pathway. Circ J 2011;75:1409-1417.

43 Li L, Chen GP, Yang Y, Ye Y, Yao L, Hu SJ: Chronic inhibition of farnesyl pyrophosphate synthase attenuates cardiac hypertrophy and fibrosis in spontaneously hypertensive rats. Biochem Pharmacol 2010;79:399406.

44 Raiteri M, Arnaboldi L, McGeady P, Gelb MH, Verri D, Tagliabue C, Quarato P, Ferraboschi P, Santaniello E, Paoletti R, Fumagalli R, Corsini A: Pharmacological control of the mevalonate pathway: effect on arterial smooth muscle cell proliferation. J Pharmacol Exp Ther 1997;281:1144-1153. 